\title{
The Study on the Image Thresholding Segmentation Algorithm
}

\author{
Yue Liu, Jia-mei Xue *, Hua Li \\ College of Information Science \& Electronic Technology, Jiamusi University, Jiamusi, 154007, \\ China \\ e-mail:liuyue197612@126.com,xuejiameixzy@163.com,jdlh65@163.com
}

${ }^{\star}$ Corresponding author

Keywords: image segmentation, threshold, Two peak algorithm, Iteration algorithm, Otsu algorithm

\begin{abstract}
Image segmentation is an important branch of image processing. Among numerous segmentation techniques, thresholding is a very important and effective one which segments different objects using a threshold. This paper discusses workings of several common thresholding segmentation methods and summarizes their respective strength and weakness from the perspective of experiments.
\end{abstract}

\section{Introduction}

Image segmentation is an essential procedure of image analysis and provides a basis for further understanding of an image. Image segmentation can be defined as a process of segmenting digital image into similar and non-overlapping divisions. The divisions can be considered as a connected set of pixels, which means a pixel set where all the pixels are adjacent or connected ${ }^{[1]}$. Image segmentation has been widely used in many fields. Figure 1 has shown some of the specific fields.

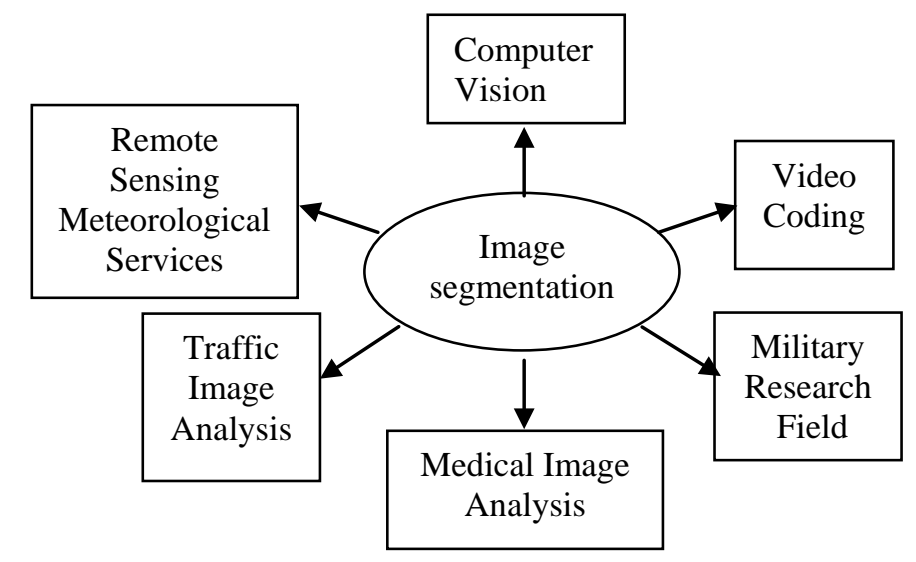

Fig.1 Application of image segmentation

As a widely used segmentation technique, thresholding makes use of the grayscale difference between the target area to be extracted and its background and finds a reasonable threshold from the two areas with different grayscale levels (target area and background area). This threshold can be used to determine whether a pixel belongs to the target area or the background area. Thus a corresponding binary image can be produced ${ }^{[2]}$.

\section{Thresholding segmentation}

There is gray scale discontinuity in boundaries between different areas in an image. In other words, the gray step change results in these boundaries. So, image segmentation can be achieved according to the gray discontinuity among pixels through algorithms finding the color or gray mutation between neighboring pixels ${ }^{[3]}$. Threshold segmentation is a method that segments an image into the background and objects according to the difference in gray value. Threshold approach is an 
image segmentation technology based on division. The basic principle of threshold segmentation is to divide image pixels into different types by setting different featured thresholds ${ }^{[4]}$. The common features include grey or colored features from original image and features transformed from original grey or colored features. Assume $\mathrm{f}(\mathrm{x}, \mathrm{y})$ as the original image, find the eigenvalue in $\mathrm{f}(\mathrm{x}, \mathrm{y})$ based on specific principle, segment the image into two parts and we can obtain the segmented image as:

$$
g(x, y)= \begin{cases}1 & f(x, y) \geq T \\ 0 & f(x, y)<T\end{cases}
$$

Which is know as the image binarization.

As for a grey image, the simplest way of thresholding is manual segmentation, by which segmentation thresholds are set by hand and different thresholds will lead to different results. However, manual segmentation is boring and time-consuming, and the segmentation results are usually inaccurate and unrepeatable ${ }^{[5]}$. So, the essential part of threshold segmentation lies in the method of choosing the threshold. Therefore, according to different threshold choosing methods, image segmentation algorithms can be subdivided into double-peak method, iterative method and Otsu's method. Next we will compare the 3 common threshold approaches.

\section{Two Peak Algorithm}

Principles of the double-peak method hold that an image is composed of the foreground and background or of two groups of colors. In a gray scale histogram, the distribution of gray scale values forms peak-like shapes. The valley between two peaks is where the threshold reside ${ }^{[6]}$. Figure 2 shows the distribution of gray values of an image. The abscissa represents while the ordinate the frequency of appearance of gray pixels in an image. The value at the valley between two peaks is used as the threshold.

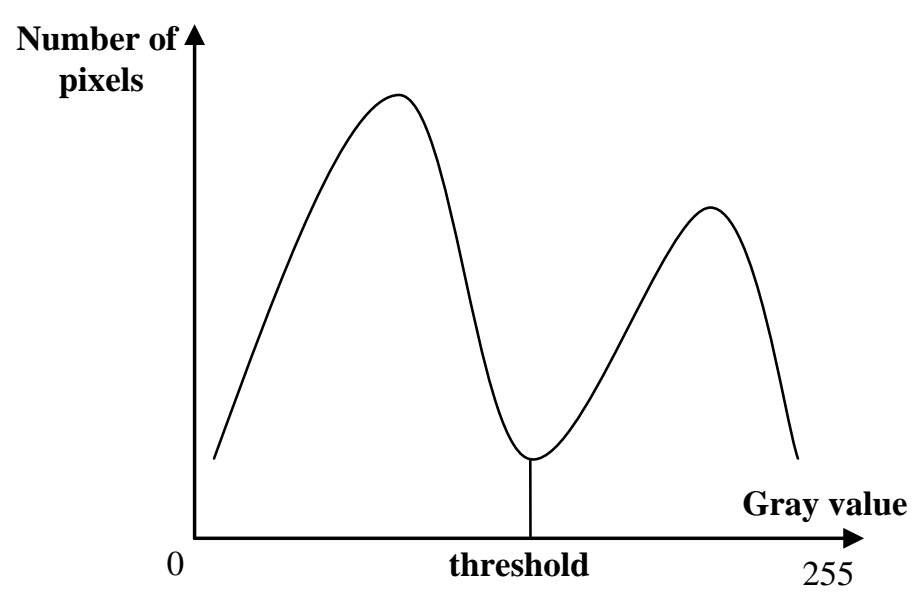

Fig. 2 Histogram

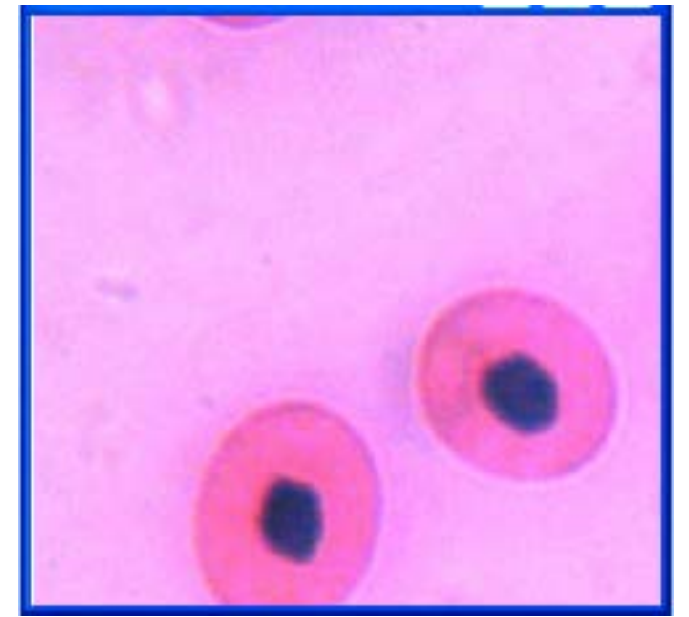

Fig. 3 original image

The workings of the two-peak method are as follows. First, input an image and gray it. Then, calculate the gray scale value of every pixel and reckon up the number of pixels at each gray scale level. Finally, calculate the values of two peaks. The minimum between the two peak values is the threshold. Figure 3 and 4 show the original image and the processed image using the double-peak method, respectively.

Existence of two peaks is a prerequisite for this histogram-based method. The two-peak method is inapplicable to images which exhibit a single peak, multiple peaks or wide, flat valley between peaks in the histogram. Figure 6 shows the histogram of Figure 5. Since there are three peaks in the histogram, the proper threshold cannot be obtained using the double-peak method. The improper choice of the threshold would fail the image segmentation as shown in Figure 7. Two-peak method is simple and direct, which can be easily used in multiple threshold segmentation occasions. However, the result of the approach is lack of objective evaluation base, which means the segmentation performance may be not the best. From the perspective of segmentation effect, the 
effect is good when the contrast between foreground and background is strong. Otherwise, it is noneffective.

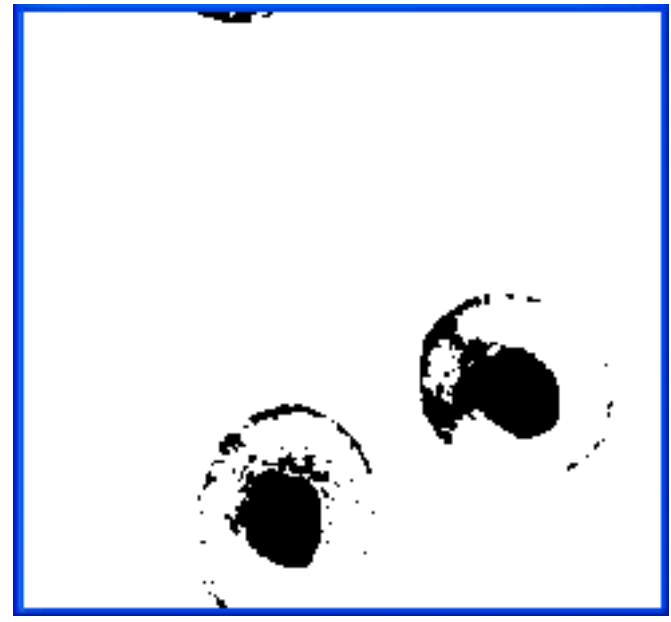

Fig. 4 Split Results in Figure

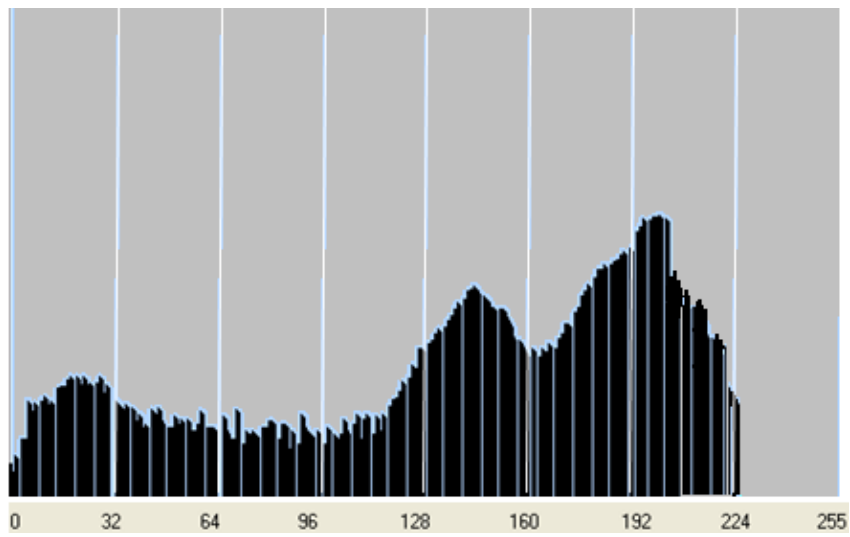

Fig. 6 Histogram

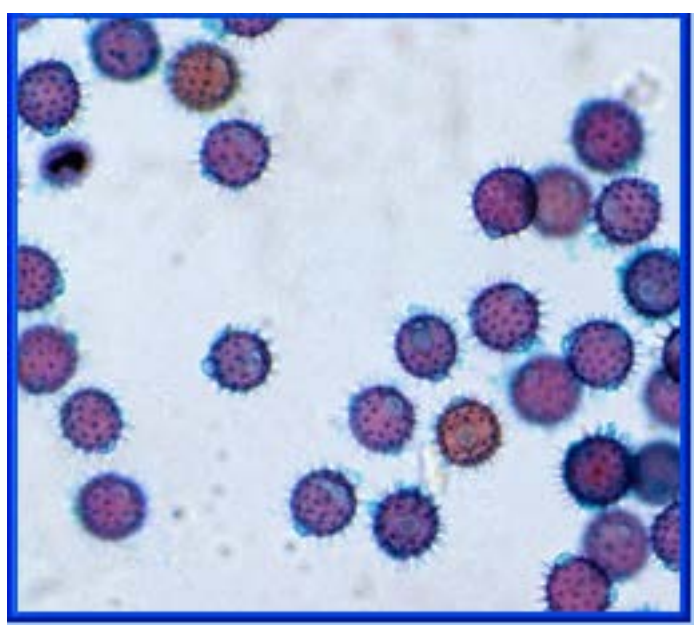

Fig. 5 original image

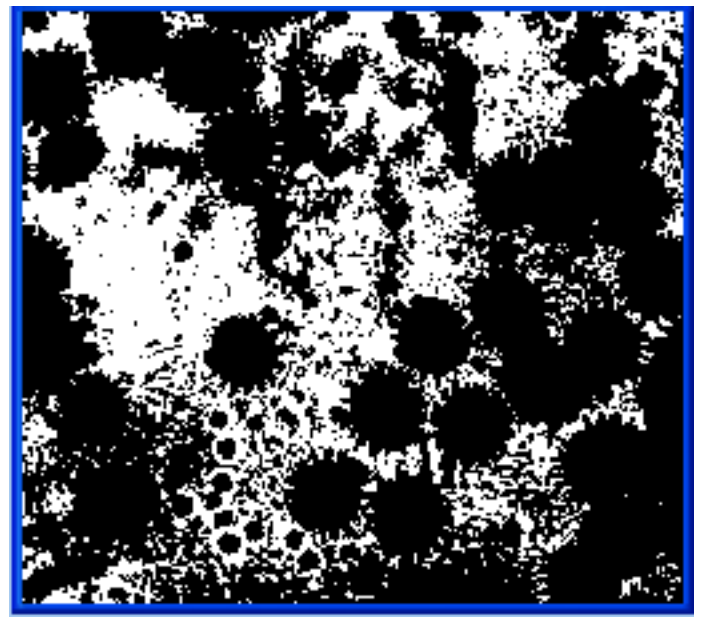

Fig. 7 Split Results in Figure

\section{Iteration Algorithm}

As for a digital image showing two peaks in its histogram, the histogram-based method can be used to find the proper threshold easily, where the valley between the two peaks is the threshold. However, it is less likely to determine the proper threshold using the double-peak method when an image has one peak, more than two peaks or no peaks at all. In this case, the iterative method is a better choice for determining the threshold. The iterative method is based the thought of approaching. Its implementation steps are as follows:

1) Find the maximum and minimum grayscale values of the image, which are denoted by ZMax and ZMin respectively. Make the initial threshold $\mathrm{T}_{0}=(\mathrm{ZMax}+\mathrm{ZMin}) / 2$.

2) Segment the image into two areas according to the threshold $T_{0}$. Calculate the average grayscale values of the two areas $\mathrm{Z} 1$ and $\mathrm{Z} 2$.

3) Calculate the new threshold $\mathrm{T}=(\mathrm{Z} 1+\mathrm{Z} 2) / 2$.

4) Specify a minimum $\varepsilon$. If $\left|\mathrm{T}-\mathrm{T}_{0}\right|<\varepsilon$, then the obtained value is the threshold and $\mathrm{T}$ is the ultimate result of the iteration. Otherwise, make $T_{0}=T$ and restart the calculation from step 2 until the error requirements are met.

Although the iterative method encompasses a larger amount of computation than the histogrambased method, it can find the optimal threshold of an image ${ }^{[7]}$. The threshold obtained using the iterative method has a good performance in terms of image segmentation by distinguishing the main 
areas of foreground and background. But this method is unable to distinguish minor areas precisely in an image. Figure 8 shows the segmentation result of Figure 2 using the iterative method.

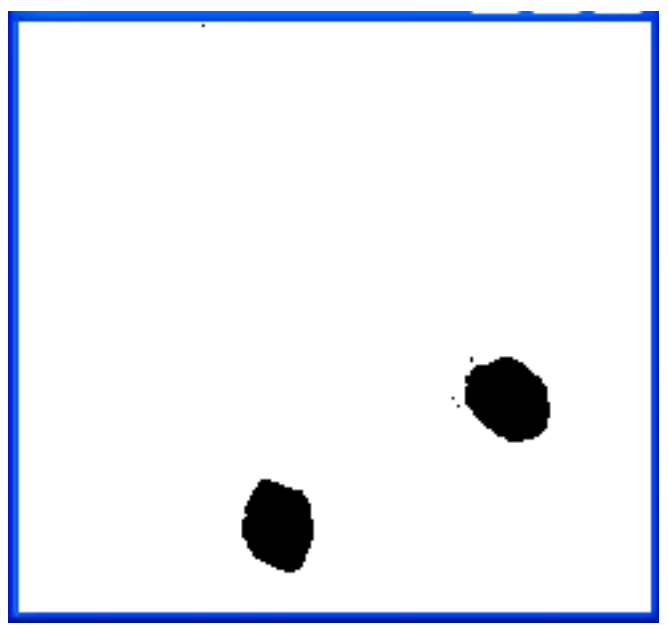

Fig. 8Split Results in Figure

\section{Otsu Algorithm}

Otsu Algorithm proposed by the Japanese researcher Nobuyuki Otsu in 1980 is an intra-class variance method $^{[8]}$. It is a simple and efficient adaptive method for computing a single threshold (for converting a grayscale image into a binary image). With Otsu Algorithm, the histogram of the grayscale image is analyzed. The histogram is segmented into two parts where the threshold is regarded as the boundary $\mathrm{T}$ moving from left in the histogram. Two groups of new segmentations are compared at a time. The optimal value of $\mathrm{T}$ is obtained from the variance of groups. When the value leads to the maximum distance of the boundary between the two parts, the demarcation point of the boundary $\mathrm{T}$ is the proper threshold. If $\mathrm{t}$ is the segmentation threshold between the foreground and background, then the ratio of foreground pixel number to total pixel number is W0, its average grayscale value is $\mathrm{U} 0$, the ratio of background pixel number to total pixel number is $\mathrm{W} 1$, its average grayscale value is $\mathrm{U} 1$. The grand average of the image is $\mathrm{U}=\mathrm{W} 0 * \mathrm{U} 0+\mathrm{W} 1 * \mathrm{U} 1$. Traverse t from the minimum to the maximum grayscale value. The optimal threshold is obtained once $t$ makes the function $\mathrm{G}=\mathrm{W} 0 *(\mathrm{U}-\mathrm{U} 0) 2+\mathrm{W} 1 *(\mathrm{U} 1-\mathrm{U}) 2$. Figure 9 shows the segmentation results using the threshold obtained by Otsu's method.

The test showed that this approach is easy, stable and effective ${ }^{[4]}$. Otsu's method would yield satisfactory results whether the histogram of an image displays obvious double peaks or not. Therefore, Otsu's method is the best choice for automatic threshold selection globally. But Otsu's method is unsuitable for handling images with a low signal-to-noise ratio.

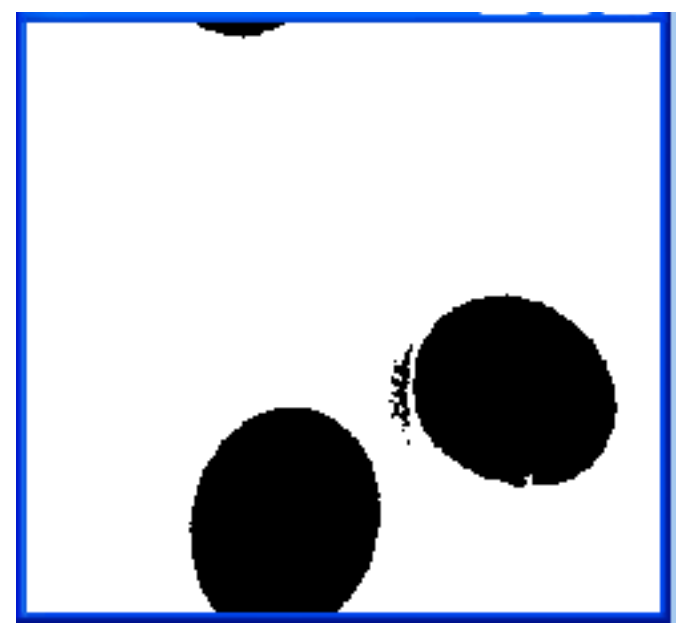

Fig. 9 Split Results in Figure 
Table 1 PERFORMANCE COMPARISON

\begin{tabular}{|c|c|c|c|c|}
\hline Threshold approaches & Applicable image & Effect & Computation & Speed \\
\hline Two peak algorithm & $\begin{array}{c}\text { Strong contrast between } \\
\text { the target and background }\end{array}$ & Preferable & Fast \\
\hline Iteration algorithm & All images & Good & Common & Common \\
\hline Otsu algorithm & $\begin{array}{c}\text { interclass variance single- } \\
\text { peak image }\end{array}$ & Preferable & Small & Fast \\
\hline
\end{tabular}

Performance Comparison

The performances of the three common threshold approaches are compared in Table 1.

\section{Conclusion}

Thresholding segmentation features the small amount of computation, which is applicable when there is a strong contrast between the target area and background. What is important is that the grayscale level of the background or objects is unitary and that closed and connected boundaries can always be obtained. This paper introduces three thresholding segmentation methods. Experiments are also conducted to show their segmentation performance. In practice, there is no method that can be universally applied to all segmentation scenarios. Sometimes the methods mentioned above need to be modified to handle some complex images. Image segmentation is a field requiring more research efforts in the days to come.

\section{Acknowledgment}

The work was financially supported by the surface project on science and technology research of the Education Department of Heilongjiang province(12521546) and the surface project on science and technology research of Jiamusi University (L2014-006) .

\section{References}

[1] Rosenfield. Connectivity in Digital Pictures[J].Journal of the ACM.1970,17(1):146-160.

[2] Wang Peizhen, Chen Weinan, Image Segmentation Based on Fuzzy Clustering and Two dimensional Thresholding [J]Journal of Image and Graphics ,Vo l. 3, 1998.

[3] Chen Ningning, Achieve and Comparison of Image Segmentation Thresholding Method[J], Computer Knowledge and Technology,Vol.7,2011.

[4] Wu Yiquan,Zhu Zhaoda. Development of threshold approach in image processing in recent 30 years. ( 1962- 1992) [J]Journal of Data Acquisition \& Processing, Vol.8,1993 : 193- 201.

[5] Tan Binbin,Research and Implementation of Image Segmentation Methods[D] NORTHEASTERN UNIVERSITY, 2006.

[6] Jiang Xiangang. Engineering Software Design of Digital Image Processing Based on Delphi [M]. Beijing: China WaterPower Press, 2006.

[7] Li Meihong, For the Fingerprint ImageThreshold with Iterative Method [J], Application of Electronic Technique,2004

[8] Li Liaoliao, Binarization Algorithm Based on Image Partition Derived from Otsu Algorithm[J], Microcomputer Information, Vol.21,2005. 